\title{
Syntactic class influences phonological priming of tip-of-the-tongue resolution
}

\author{
LISE ABRAMS and EMILY L. RODRIGUEZ \\ University of Florida, Gainesville, Florida
}

\begin{abstract}
During tip-of-the-tongue (TOT) experiences, phonologically related words have both facilitated and impeded word retrieval. In the present experiment, we examined whether phonologically related words' syntactic class (part of speech) is responsible for these differential effects. Sixty college students saw general knowledge questions whose answers were designated target words and responded "know," "don't know," or "TOT." Following “TOT" and "don't know" responses, the participants saw five words, one of which was a prime. The primes contained the target's first syllable and either shared or did not share the target's part of speech. Following presentation of the primes, retrieval of the target was attempted again. Different-part-of-speech primes facilitated resolution of TOT states, whereas same-part-of-speech primes had no effect, relative to phonologically unrelated words. These results support node structure theory's most-primed-wins principle and the transmission deficit model account of TOT states and detail the importance of syntactic class in the selection of words that are candidates for speech production.
\end{abstract}

A tip-of-the-tongue (TOT) state is a well-known phenomenon that consists of a temporary inability to retrieve a known word (e.g., R. Brown \& McNeill, 1966). This frustrating experience has motivated considerable research in the past 25 years and has led to the development of several theories regarding why TOT states occur. One of the predominant theoretical explanations is the transmission deficit (TD) model, which proposes that TOT states are caused by weakened connections between a word and its phonology, or sounds (e.g., Burke, MacKay, Worthley, \& Wade, 1991; MacKay \& Burke, 1990). The TD model therefore suggests a solution for resolving TOT states: Strengthening the weak phonological links will increase retrieval of the missing word. In previous research, this prediction has been tested by inducing TOT states, presenting words containing various phonological features of the unretrieved word, and then asking participants to attempt word retrieval again. These studies showed that phonologically related words presented during TOT states increase word retrieval (e.g., Abrams, White, \& Eitel, 2003; James \& Burke, 2000; White \& Abrams, 2002).

However, phonologically related words can also inhibit or delay TOT resolution. TOT states are often ac-

This article is based on an undergraduate senior thesis written by E.L.R. Portions of this research were reported at the 16th annual convention of the American Psychological Society in May 2004 and at the 45th Annual Meeting of the Psychonomic Society in November 2004. The research was partially supported by the University of Florida University Scholars Program, an undergraduate research award granted to E.L.R. We thank Katherine White for her helpful comments on a draft of this article. Correspondence concerning this article should be sent to L. Abrams, Department of Psychology, University of Florida, P.O. Box 112250, Gainesville, FL 32611-2250 (e-mail: abrams@ufl.edu). companied by blockers, or persistent alternates - that is, incorrect words that come to mind involuntarily and typically share phonological features with the unretrieved word (e.g., MacKay \& Burke, 1990). Research has demonstrated that when TOT states are accompanied by an alternate word, TOT states are less likely to be resolved and that, even when resolved, retrieving the intended word takes longer, relative to TOT states that occur without alternate words in mind (Burke et al., 1991). These results are consistent with an inhibition model of TOT states, in which TOT states are caused by an alternate word, which comes to mind first and suppresses retrieval of the desired word (e.g., Jones, 1989).

The present experiment attempted to resolve these paradoxical findings by testing a specific hypothesis unique to node structure theory (NST; MacKay, 1987) and the TD model. This hypothesis predicts that a word's syntactic class plays a pivotal role in determining the impact that phonologically related words will have on resolution of TOT states. Specifically, Burke et al. (1991) suggested that "for subjects in the TOT state, presenting a word that is phonologically related and in a different domain (syntactic class) from the target will facilitate resolution, whereas a phonologically related word in the same domain as the target will delay resolution" (p. 570). To understand how this prediction is derived, a brief overview of NST and the TD model is given below.

NST arranges conceptual representations, or nodes, into a hierarchical network of multilevel systems, including semantic, syntactic, and phonological systems. Within this theory, word retrieval occurs as a function of two processes: node priming and node activation. Node priming is a subthreshold excitation that spreads between connected nodes, whereas node activation is the point at which a 
node receives enough priming to enable retrieval. As a corollary of NST, the TD model suggests that TOT states occur when a word's lexical node is activated but connections to the word's phonological nodes are weakened (due to infrequent or nonrecent use). The result of this weakening of connections is that the lexical node is unable to transmit sufficient node priming to enable activation of the phonology and achieve word retrieval. Similar explanations for the cause of TOT states have been proposed by other interactive models of speech production (see, e.g., Dell, 1986), as well as by discrete two-stage theories that characterize a TOT state as a failure to retrieve the phonological word form following successful lemma retrieval (see, e.g., Levelt, 1989).

The role of syntactic class in TOT state resolution is detailed by the most-primed-wins principle (Burke et al., 1991; MacKay, 1987; MacKay \& Burke, 1990), which states that when nodes in the same syntactic domain receive simultaneous priming, only the node receiving the most priming can be activated at any given point in time. In other words, if a phonologically related word is activated and is the same part of speech as the unretrieved TOT word, the phonologically related word's activation level must subside before the TOT word can be activated, delaying resolution of the TOT state. In contrast, presenting a phonologically related word whose part of speech is different will not interfere with retrieval of the TOT word. In fact, a phonologically related word will facilitate retrieval of the TOT word by transmitting bottom-up priming to other lexical nodes connected to it. Since the TOT word is connected phonologically to the related word and is in another syntactic domain, it will then accumulate enough priming to become activated and will be retrieved (see MacKay \& Burke, 1990, and Burke et al., 1991, for more details on the theory and these predictions).

NST and the TD model also predict that the frequency of phonologically related words presented during TOT states will interact with the proposed syntactic category effects. The lexical node for a high-frequency word has stronger connections to its phonological nodes, enabling it to remain activated for a longer period of time. Thus, a high-frequency, phonologically related word that shares syntactic domain with the TOT word should decrease resolution of the TOT state more than does a low-frequency word, because the competing word remains activated longer, preventing activation of the target. Conversely, a high-frequency, phonologically related word in a different syntactic domain is expected to increase resolution of the TOT state, because of the stronger transmission of priming to its connected nodes, some of which are the target's firstsyllable phonology, which should then achieve sufficient priming for activation of the entire target. Providing support for this prediction independently of syntactic domain, Vitevitch and Sommers (2003, Experiment 3) showed that target words with high neighborhood frequency (the mean frequency of all of the words that phonologically resembled the target) were produced more quickly and accurately than targets with low neighborhood frequency in a picture-naming task. Whether phonological inhibition effects can be obtained as a function of frequency (and/or syntactic class) has yet to be demonstrated.

The predictions made by NST and the TD model are not easily derived from other theories. Theories that view the TOT state as a metacognitive phenomenon (e.g., Schwartz, 1999) do not detail the relationships between semantics, syntax, and phonology, making these theories unable to generate predictions about TOT state resolution as a function of syntactic class. Furthermore, whereas other theories of language production (e.g., Dell, 1986; Levelt, 1989) explain the etiology of TOT states, these theories do not clearly specify a mechanism for detailing when facilitative versus inhibitory effects will occur in TOT state resolution or, in some cases, in speech production more generally. Using NST and the TD model, in the present experiment, we attempted to precisely specify the conditions under which phonologically related words would delay or, possibly, inhibit TOT state resolution experimentally, as do persistent alternates for naturally occurring TOT states.

\section{METHOD}

\section{Participants}

Sixty participants were recruited from undergraduate classes at the University of Florida. All were between the ages of 17 and 21 years (41 female, 19 male, mean age 18.8 years, $S D=1.0$ ) and either were native English speakers or had experience speaking English most of their lives.

\section{Materials}

An attempt to induce a TOT state occurred through presentation of a definition-like question eliciting a target word. Seventy-nine of the 96 questions were chosen from previous studies (e.g., Burke et al., 1991; Jones, 1989), and 17 new questions and targets were created. All the target words were two to five syllables in length, low in Francis and Kučera (1982) frequency (all were 0-13 per million, except one at 21 per million and one at 42 per million), and none was a proper name.

For each target word, two phonological primes were created: one that was the same part of speech as the target and one that was a different part of speech. A phonological prime contained the same first syllable as the target but did not overlap in phonology with the rest of the word, whenever possible. ${ }^{1}$ None of the primes contained a word that was another part of speech within it; for example, acrobatic was not used because it contains the entire phonology of acrobat. In addition to the primes, a phonologically unrelated word was created for each target as a control. These words shared syntactic class with the target half the time. Both the primes and the control word contained the same number of syllables. Across all stimuli, the word frequencies of the primes and the control words were relatively similar: The frequencies of same-part-of-speech primes ranged from 0 to 275 $(M=23.8, S D=46.8)$; the frequencies of different-part-of-speech primes ranged from 0 to $244(M=19.1, S D=38.8)$; and unrelated controls' frequencies ranged from 0 to $297(M=38.4, S D=62.3)$. Example stimuli are shown in Table 1.

Following each question, the participant saw a five-word list containing a prime that shared part of speech with the target, a prime that did not share part of speech, or an unrelated control word intermixed with four unrelated filler words. The prime and control words were presented as the second, third, or fourth word in the list and were equally distributed across the positions. None of the filler words in each presented list began with the same letter, and 
Table 1

\begin{tabular}{|c|c|c|c|c|}
\hline & Target & $\begin{array}{c}\text { Same- } \\
\text { Part-of-Speech } \\
\text { Prime }\end{array}$ & $\begin{array}{c}\text { Different- } \\
\text { Part-of-Speech } \\
\text { Prime }\end{array}$ & $\begin{array}{l}\text { Unrelated } \\
\text { Control } \\
\text { Word }\end{array}$ \\
\hline Word & intransitive & incredible & insecticide & dictionary \\
\hline Part of speech & adjective & adjective & noun & noun \\
\hline Frequency & 0 & 23 & 3 & 59 \\
\hline $\begin{array}{l}\text { Word } \\
\text { Part of speech }\end{array}$ & $\begin{array}{l}\text { canonize } \\
\text { verb }\end{array}$ & $\begin{array}{l}\text { cancel } \\
\text { verb }\end{array}$ & $\begin{array}{l}\text { candid } \\
\text { adiective }\end{array}$ & $\begin{array}{l}\text { hectic } \\
\text { adiective }\end{array}$ \\
\hline Frequency & 2 & 17 & 3 & 3 \\
\hline Word & rosary & robot & robust & fever \\
\hline Part of speech & noun & noun & adjective & noun \\
\hline Frequency & 3 & 4 & 0 & 19 \\
\hline Word & actuary & acrobat & accurate & stimuli \\
\hline Part of speech & noun & noun & adjective & noun \\
\hline Frequency & 0 & 1 & 35 & 0 \\
\hline
\end{tabular}

no filler word shared any phoneme with its corresponding target or overlapped in first-syllable phonology with any other target. In each list, none of the words had an obvious semantic relationship to any of the others or to the target. ${ }^{2}$

The experiment was run on Pentium II, 350-MHz PC-compatible computers using a program written in Visual Basic 5.0.

\section{Design and Procedure}

In the experiment, a single-factor design was used, with prime condition (same-part-of-speech primes, different-part-of-speech primes, and unrelated controls) as a within-participants factor. ${ }^{3}$ The participant was given written and verbal instructions describing a TOT state as the certainty of knowing the correct answer, accompanied by a feeling of not quite being able to retrieve it. A general knowledge question was then presented, in random order, with instructions at the bottom of the screen to say "know," "don't know," or "TOT." Following a "don't know" or "TOT" response, the list of four filler words intermixed with a word from one of the three prime conditions was presented one word at a time, centered on the screen. If the participant initially responded "know," he or she was prompted to give the answer and then saw a list of five words that always contained a control word.

To disguise the relation between the primes and the targets, the participant was told that the word lists were relevant to an unrelated study involving people's knowledge about words' grammar and frequency of occurrence. After presentation of each word, the participant was instructed to verbally indicate its part of speech (noun, verb, adjective, or other), as well as the frequency with which he or she had encountered the word in everyday life, using a scale of 1 (never having encountered the word) to 7 (on a daily basis). After these ratings had been completed for all five words, the participant saw either the next question (if he or she had initially responded "know") or the same question again (if he or she had initially responded "don't know" or "TOT"). If the participant now knew the answer, he or she verbally stated the word. After all 96 questions had been presented, a recognition test was administered to determine whether or not the participant had experienced unresolved TOT states for the intended target. This test consisted of the questions for which the participant never retrieved an answer, along with four possible answers to choose from: the target, a word phonologically similar to the target, a word semantically related to the target, and a word unrelated to the target. Following the recognition test, a postexperiment questionnaire was administered verbally to assess the participant's awareness of the phonological relationship between the primes and the targets, as well as intentional use of that knowledge to aid in target retrieval. The experimenter was responsible for typing in all responses, and all sessions were recorded on cassette tape.

\section{RESULTS}

\section{Postexperiment Questionnaire}

The responses on the postexperiment questionnaire revealed that only 1 participant was aware that some of the primes contained the same letters or sounds as the targets; however, this participant reported being unable to intentionally use this knowledge during retrieval attempts and, therefore, was not excluded from analyses. One participant was excluded from all the analyses because of recent participation in an experiment in which similar general knowledge questions and targets had been used.

\section{Initial Responses}

For initial responses to the general knowledge questions, $41 \%$ were "don't know" responses, $34.5 \%$ were correct "know" responses, and $8 \%$ were correct "TOT" responses. A response was considered correct if the participant produced the correct answer after an initial or second "know" response or if he or she chose the correct answer for an unresolved TOT state on the recognition test. Incorrect "know" responses (13.8\%) and incorrect TOT responses $(2.7 \%)$ were excluded from all statistical analyses.

\section{Target Retrieval Following "TOT" Responses}

Because the control words shared syntactic class with the target only half the time, a paired samples $t$ test was conducted on TOT state resolution following lists containing control words. Lists containing controls that were the same part of speech as the target were compared with controls that were a different part of speech. Twenty-three participants could not be included in this analysis because they did not have any correct "TOT" responses preceding both types of control lists. The $t$ test was not significant $[t(35)=0.184, p>.10]$, since TOT state resolution following presentation of same-part-of-speech control words $(M=27.3 \%)$ was equivalent to TOT state resolution following presentation of different-part-of-speech control words $(M=28.9 \%)$. Therefore, the two types of control word lists were collapsed into a single unrelated condition for the remaining analyses. 
Table 2

Target Word Retrieval (in Percentages) Following Primes and Unrelated Control Words After an Initial "TOT" or "Don't Know" Response

\begin{tabular}{|c|c|c|c|c|c|}
\hline \multirow[b]{3}{*}{ Initial Response } & \multirow[b]{3}{*}{ Priming Condition } & \multicolumn{4}{|c|}{ Retrieval } \\
\hline & & \multicolumn{2}{|c|}{$\begin{array}{c}\text { Participant } \\
\text { Analysis } \\
\end{array}$} & \multicolumn{2}{|c|}{$\begin{array}{c}\text { Item } \\
\text { Analysis }\end{array}$} \\
\hline & & $M$ & $S D$ & $M$ & $S D$ \\
\hline \multirow[t]{3}{*}{ TOT } & Same-part-of-speech primes & 26.0 & 28.6 & 23.1 & 33.2 \\
\hline & Different-part-of-speech primes & 37.1 & 37.6 & 30.2 & 36.5 \\
\hline & Unrelated control words & 25.0 & 28.6 & 22.0 & 35.3 \\
\hline \multirow[t]{3}{*}{ Don’t know } & Same-part-of-speech primes & 5.2 & 7.4 & 5.6 & 11.1 \\
\hline & Different-part-of-speech primes & 5.2 & 6.8 & 6.6 & 13.0 \\
\hline & Unrelated control words & 4.7 & 7.3 & 5.6 & 11.2 \\
\hline
\end{tabular}

For "TOT" responses, the mean percentage of target retrieval and the standard deviations following each priming condition are displayed in the top half of Table 2. A single-factor, repeated measures ANOVA was performed on percentage of correct retrieval of the target. This analysis was performed by participants and by items. Nine participants $(15 \%)$ were unable to be included in the ANOVA, as were 52 items (54\%), for not having at least one correct "TOT" response for each of the three levels of prime condition. The effect of prime condition was significant in the participant analysis only $\left[F_{1}(2,98)=3.24\right.$, $\left.M S_{\mathrm{e}}=699.9, p<.05 ; F_{2}<1\right]$, although the trend in the item analysis was in the same direction as that in the participant analysis. ${ }^{4}$ Post hoc tests on the participant analysis revealed significant priming $(p<.05)$, so that there was greater retrieval of the target following different-part-ofspeech primes, relative to unrelated control words. Target retrieval following different-part-of-speech primes was also greater than retrieval following same-part-of-speech primes. In contrast, resolution of TOT states was equivalent for same-part-of-speech primes and unrelated words $(p>.10)$. To increase power in the item analysis in order to detect significant priming for different-part-of-speech primes, pairwise comparisons were conducted between each type of prime and the unrelated control words. Paired sample $t$ tests revealed marginally significant priming for different-part-of-speech primes $[t(53)=1.92, p<.06]$, but not for same-part-of-speech primes $[t(57)=0.52, p>$ $.10]$, in agreement with the participant analysis.

\section{Target Retrieval Following "Don't Know" Responses}

To examine whether the priming effect above was unique to TOT state resolution, the same repeated measures ANOVAs were conducted for percentage of correct retrieval of targets following "don't know" responses. The means and standard deviations are displayed in the bottom half of Table 2. Although no participants were eliminated from the participant analysis, four items (4\%) were unable to be included for not having a "don't know" response for each of the three levels of prime condition. The ANOVA revealed no effect of prime condition in either the participant analysis $\left(F_{1}<1\right)$ or the item analysis $\left(F_{2}<1\right)$.

\section{Correlations Between Prime Frequency and Target Retrieval}

To assess whether prime frequency influenced TOT state resolution, Pearson correlations were computed between the primes' Francis and Kučera (1982) frequency ratings and the percentages of correct target retrieval following "TOT" responses. For same-part-of-speech primes, there was a significant negative correlation $[r(150)=-.18$, $p<.05$ ], whereas there was a significant positive correlation for different-part-of-speech primes $[r(147)=.17$, $p<.05]$. In contrast, there was no significant correlation for unrelated controls $[r(154)=.09, p>.10]$.

\section{Correlations Between Prime Position and Target Retrieval}

Because the prime was presented in one of three list positions (second, third, or fourth word), it was possible that the prime's position influenced TOT state resolution. Given the small number of "TOT" responses, it was not feasible to conduct an ANOVA with list position as an additional factor; however, Pearson correlations were computed between the primes' position and the percentage of correct target retrieval following "TOT” responses. For same-partof-speech primes, there was a significant positive correlation $[r(151)=.20, p<.05]$, whereas there was no significant correlation for either different-part-of-speech primes $[r(148)=-.07, p>.10]$ or unrelated controls $[r(154)=$ $-.09, p>.10]$. Means and standard deviations for each prime type and list position are shown in Table 3.

Table 3

Target Word Retrieval (in Percentages) After an Initial "TOT" Response as a Function of Prime Type and List Position

\begin{tabular}{|c|c|c|c|}
\hline \multirow{2}{*}{ Priming Condition } & \multirow[b]{2}{*}{ List Position } & \multicolumn{2}{|c|}{ Retrieval } \\
\hline & & $M$ & $S D$ \\
\hline \multirow[t]{3}{*}{ Same-part-of-speech primes } & Second position & 17.5 & 38.5 \\
\hline & Third position & 21.3 & 41. \\
\hline & Fourth position & 40.0 & 49. \\
\hline \multirow[t]{3}{*}{ Different-part-of-speech primes } & Second position & 36.6 & 48. \\
\hline & Third position & 33.3 & 47 \\
\hline & Fourth position & 28.0 & 45. \\
\hline \multirow[t]{3}{*}{ Unrelated control words } & Second position & 33.3 & 47. \\
\hline & Third position & 23.5 & 42 \\
\hline & Fourth position & 22.0 & 41 \\
\hline
\end{tabular}




\section{DISCUSSION}

The results demonstrate that presentation of phonologically related words does not always facilitate TOT state resolution. As is predicted by NST and the TD model (e.g., Burke et al., 1991; MacKay, 1987; MacKay \& Burke, 1990), the syntactic class of phonologically related words played a significant role, where only different-part-ofspeech primes facilitated TOT state resolution, relative to unrelated words. These results are consistent with other findings of phonological priming of TOT state resolution (e.g., Abrams et al., 2003; James \& Burke, 2000; White \& Abrams, 2002) and extend earlier studies by illustrating that only a single presentation of a prime is needed to increase word retrieval, supporting the TD model's idea that recent presentation of words serves to strengthen connections to their phonological nodes. Furthermore, this phonological priming effect occurred only following "TOT" responses; retrieval of targets following "don't know" responses did not benefit from presentation of any phonologically related word, which is also consistent with previous research (James \& Burke, 2000; White \& Abrams, 2002).

In contrast, presenting same-part-of-speech primes had no effect on TOT state resolution, such that resolution of TOT states following same-part-of-speech primes was equivalent to TOT state resolution following unrelated words, despite the shared phonology between the primes and the targets. Consistent with the predictions of NST and the TD model, same-part-of-speech primes "delayed" TOT state resolution, resulting in no increased resolution, relative to unrelated words. Interestingly, same-part-of-speech primes did not inhibit TOT state resolution, which would have resulted in fewer TOT states resolved, relative to unrelated words. To achieve inhibition, a same-part-of-speech prime must be a strong competitor of the target, which will enable the competitor to maintain its activation level for a longer period of time and result in inhibition of TOT state resolution. The correlation analyses provide some evidence of factors that may lead to an inhibitory effect in TOT state resolution for same-part-of-speech primes: high word frequency and early list position. The Francis and Kučera (1982) frequency of the same-part-of-speech primes negatively correlated with TOT state resolution. This correlation indicates that more frequent same-part-of-speech primes were less likely to lead to TOT state resolution, as is predicted by the TD model (e.g., MacKay \& Burke, 1990). Theoretically, high-frequency primes take more time to decrease their activation levels, which must happen before the target can be activated, leading to a reduction in retrieval of the target. By virtue of weaker connections, low-frequency primes can become deactivated relatively quickly, making them less competitive with the target for retrieval. The majority of same-part-of-speech primes in this experiment were low frequency (i.e., $75 \%$ had a frequency less than 20 ), which may have also contributed to same-part-of-speech primes not inhibiting TOT state resolution overall. Another possibility is that some high-frequency primes produced inhibition but that the inhibition effect was canceled out by low-frequency primes that produced facilitation.
A prime's position in the word list also significantly correlated with TOT state resolution, but positively: The earlier in the list the same-part-of-speech prime occurred, the less likely the TOT state was to be resolved. This finding is important because it suggests that for a same-partof-speech prime to compete with the TOT word, it must be presented quickly after the TOT state occurs. Within NST and the TD model, some of the TOT word's phonological nodes may receive sufficient priming to become activated, even if the entire word cannot be retrieved. The activated phonological nodes then transmit priming bottom up to all lexical nodes containing this phonology. Thus, as the interval between the onset of the TOT state and the presentation of the prime increases (e.g., by presenting the prime later in the list), there is a greater likelihood that the bottom-up priming transmitted from the activated phonological nodes will have accumulated on a phonologically related word in a different syntactic domain, resulting in facilitation and making the same-part-of-speech prime less able to accumulate the most node priming to become a competitor. In contrast, early presentation of a samepart-of-speech prime allows the prime to quickly become the most primed word in its syntactic domain, preventing activation of the target and delaying TOT state resolution. These findings cannot be explained by two-stage theories of language production (e.g., Levelt, 1989), which do not have a mechanism for allowing activation to spread "backward" from the phonology to the word-form level.

In contrast to same-part-of-speech primes, a differentpart-of-speech prime's frequency and prime position had the alternative relationship with TOT state resolution. The frequency of different-part-of-speech primes positively correlated with TOT state resolution, consistent with the idea that primes outside of the target's domain can facilitate retrieval by transmitting node priming to phonologically related words, one of which is the target. Despite their opposite directions, the correlations for same- and different-part-of-speech primes were equivalent in magnitude, suggesting that frequency's influence was of similar strength in both conditions. Unlike word frequency, a prime's serial position had no relationship with TOT state resolution for different-part-of-speech primes. Since the primes are in a different syntactic class, they are not competitors with the TOT word for retrieval, making the timing of their presentation irrelevant to TOT resolution.

In sum, the present experiment resolves a debate that has existed for decades: Do phonologically related words facilitate or impede TOT state resolution? The answer is that part of speech mediates the relationship between phonological priming and word retrieval during TOT states, a finding that is of considerable importance in understanding when blocking effects do and do not occur in speech production. The results of the present experiment also speak more generally to the role of syntactic class and its relevance to lexical retrieval in speech production. Syntactic class activates words that are candidates for production, so that phonologically similar words within the same syntactic class compete with each other, whereas phonologically similar words in different syntactic classes facili- 
tate word retrieval. This finding supports the existence of a fundamental syntactic mechanism for language production proposed within NST, where word order is controlled by activating only those words that are in the appropriate syntactic class.

\section{REFERENCES}

Abrams, L., White, K. K., \& Eitel, S. L. (2003). Isolating phonological components that increase tip-of-the-tongue resolution. Memory \& Cognition, 31, 1153-1162.

Brown, A. S. (1991). A review of the tip-of-the-tongue experience. Psychological Bulletin, 109, 204-223.

Brown, R., \& McNeILL, D. (1966). The "tip of tongue" phenomenon. Journal of Verbal Learning \& Verbal Behavior, 5, 325-337.

Burke, D. M., MacKay, D. G., Worthley, J. S., \& Wade, E. (1991). On the tip of the tongue: What causes word finding failures in young and older adults? Journal of Memory \& Language, 30, 542-579.

DeLL, G. S. (1986). A spreading-activation theory of retrieval in sentence production. Psychological Review, 93, 283-321.

Francis, W. N., \& KuČERA, H. (1982). Frequency analysis of English usage: Lexicon and grammar. Boston: Houghton Mifflin.

James, L. E., \& Burke, D. M. (2000). Phonological priming effects on word retrieval and tip-of-the-tongue experiences in young and older adults. Journal of Experimental Psychology: Learning, Memory, \& Cognition, 26, 1378-1391.

Jones, G. V. (1989). Back to Woodworth: Role of interlopers in the tipof-the-tongue phenomenon. Memory \& Cognition, 17, 69-76.

Levelt, W. J. M. (1989). Speaking: From intention to articulation. Cambridge, MA: MIT Press.

MacKAY, D. G. (1987). The organization of perception and action: A theory for language and other cognitive skills. New York: Springer.

MacKaY, D. G., \& BURKe, D. M. (1990). Cognition and aging: A theory of new learning and the use of old connections. In T. Hess (Ed.), Aging and cognition: Knowledge organization and utilization (pp. 213-263). Amsterdam: North-Holland.

Schwartz, B. L. (1999). Sparkling at the end of the tongue: The etiology of tip-of-the-tongue phenomenology. Psychonomic Bulletin \& Review, 6, 379-393.

ViteVitch, M. S., \& Sommers, M. S. (2003). The facilitative influence of phonological similarity and neighborhood frequency in speech production in younger and older adults. Memory \& Cognition, 31, 491-504.

White, K. K., \& Abrams, L. (2002). Does priming specific syllables during tip-of-the-tongue states facilitate word retrieval in older adults? Psychology \& Aging, 17, 226-235.

\section{NOTES}

1. Abrams et al. (2003) showed that a single-phoneme prime was not sufficient for priming TOT state resolution. Therefore, for six targets whose first syllable was a single phoneme, we included the next phoneme in both primes; for example, for the target atone, the primes were constructed to include the first phoneme of the second syllablethat is, attenuate and atrocity. Most important, same-part-of-speech primes and different-part-of-speech primes contained the same amount of overlap for these targets.

2 . To ensure that the primes in the same syntactic class as the target were no more semantically related to the targets than were the primes in a different syntactic class, 8 additional participants (who were not in the actual experiment) were shown the targets paired with the corresponding same-part-of-speech prime, as well as the targets paired with the different-part-of-speech prime. All the participants rated both pairs, but half rated the same-part-of-speech primes first, and the other half rated the different-part-of-speech primes first. The participants were instructed to "think carefully about the meanings of both words" and to rate each pair of words on a scale from 1 (not at all related) to 7 (identical in meaning) in terms of the words' overlap in their meanings. A paired samples $t$ test compared the mean ratings for the two types of primes and showed no significant difference between the targets' semantic relation to same-part-of-speech primes $(M=1.19)$ and different-part-of-speech primes $[M=1.14 ; t(7)=1.49, p>.10]$, with both types of primes demonstrating virtually no semantic relation to the targets.

3. Prime frequency was not manipulated as a variable, because TOT states occur on a relatively small proportion of trials in experimental research (approximately $8 \%-18 \%$ of retrieval attempts; A. S. Brown, 1991). A 3 (prime condition) $\times 2$ (prime frequency) design would have resulted in only one or two correct "TOT" responses in each of the six cells, which would increase within-participants variability and reduce statistical power (see also R. Brown \& McNeill, 1966, and Burke et al., 1991).

4. Item analyses in TOT research are not typically reported, because many stimuli are eliminated due to insufficient TOT states in all conditions, resulting in low statistical power for detecting significance in item analyses.

(Manuscript received July 2, 2004; revision accepted for publication March 26, 2005.) 